This item was submitted to Loughborough's Research Repository by the author.

Items in Figshare are protected by copyright, with all rights reserved, unless otherwise indicated.

\title{
Stability of shear shallow water flows with free surface
}

PLEASE CITE THE PUBLISHED VERSION

https://doi.org/10.1137/16M1098164

\section{PUBLISHER}

(c) Society for Industrial and Applied Mathematics

\section{VERSION}

NA (Not Applicable or Unknown)

\section{PUBLISHER STATEMENT}

This work is made available according to the conditions of the Creative Commons Attribution-NonCommercialNoDerivatives 4.0 International (CC BY-NC-ND 4.0) licence. Full details of this licence are available at: https://creativecommons.org/licenses/by-nc-nd/4.0/

\section{LICENCE}

CC BY-NC-ND 4.0

\section{REPOSITORY RECORD}

Chesnokov, A.A., G.A. El, S.L. Gavrilyuk, and Maxim V. Pavlov. 2019. "Stability of Shear Shallow Water Flows with Free Surface". figshare. https://hdl.handle.net/2134/24313. 


\title{
STABILITY OF SHEAR SHALLOW WATER FLOWS WITH FREE SURFACE*
}

\author{
A. A. CHESNOKOV ${ }^{\dagger}$, G. A. EL ${ }^{\ddagger}$, S. L. GAVRILYUK ${ }^{\S}$, AND M. V. PAVLOV
}

\begin{abstract}
Stability of inviscid shear shallow water flows with free surface is studied in the framework of the Benney equations. This is done by investigating the generalized hyperbolicity of the integrodifferential Benney system of equations. It is shown that all shear flows having monotonic convex velocity profiles are stable. The hydrodynamic approximations of the model corresponding to the classes of flows with piecewise linear continuous and discontinuous velocity profiles are derived and studied. It is shown that these approximations possess Hamiltonian structure and a complete system of Riemann invariants, which are found in an explicit form. Sufficient conditions for hyperbolicity of the governing equations for such multilayer flows are formulated. The generalization of the above results to the case of stratified fluid is less obvious, however, it is established that vorticity has a stabilizing effect.
\end{abstract}

Key words. free surface flows, shallow water waves, shear flows, hydrodynamic stability, hyperbolicity

AMS subject classifications. 76E05, 35L60, 76B15

DOI. $10.1137 / 16 \mathrm{M} 1098164$

1. Introduction. The classical shallow water equations [22] describe the propagation of long waves on a free surface under the assumption that the flow under consideration is potential. In this model, only the depth-averaged velocities are used in the formulation of the governing equations. However, in practice, fluid flows are sheared, which is mainly due to the viscosity effects near boundaries. Obviously, for a more accurate modeling of the wave propagation it is also necessary to take into account nonuniformity of the flow.

An extension of the classical shallow water theory to vortical fluid flows was proposed by Burns [3], who was the first to study plane parallel shear flows of an inviscid fluid in linear approximation and derive the dispersion relation for normal modes. In this case, the speed of propagation of a perturbation is determined by an integral relation depending on the horizontal velocity profile over depth. A nonlinear model of long surface waves in shear flow was derived by Benney [1] and represents an integro-differential system of equations in contrast with the quasi-linear system for potential flow. Nevertheless, it was shown in [1] that the Benney system can be written in the form of the so called infinite-component moment chain and, similarly to the classical shallow water equations, possesses infinitely many conservation laws. Zakharov [28] established the equivalence between the Benney system and the Vlasov kinetic equation, and Teshukov, Russo, and Chesnokov [25] found an explicit transformation between these two models. Kupershmidt and Manin [16] and Lebedev and

*Received by the editors October 10, 2016; accepted for publication (in revised form) February 10, 2017; published electronically June 29, 2017.

http://www.siam.org/journals/siap/77-3/M109816.html

Funding: This work was supported by the Russian Science Foundation (grant 15-11-20013).

${ }^{\dagger}$ Novosibirsk State University, and Lavrentyev Institute of Hydrodynamics SB RAS, Novosibirsk 630090, Russia (chesnokov@hydro.nsc.ru).

${ }^{\ddagger}$ Centre for Nonlinear Mathematics and Applications, Department of Mathematical Sciences, Loughborough University, Loughborough LE11 3TU, United Kingdom (g.el@lboro.ac.uk).

§Aix-Marseille Université, UMR CNRS 7343, IUSTI, 13453 Marseille CEDEX 13, France (sergey.gavrilyuk@univ-amu.fr).

『Novosibirsk State University, Novosibirsk 630090, Russia (mpavlov@itp.ac.ru).

1068 
Manin [17] found the local Hamiltonian structure and the Lax pair, respectively, for the Benney system. Families of exact solutions, having the structures of traveling and simple waves, were constructed and interpreted by Freeman [12], Sachdev [20], Varley and Blythe [27] and Teshukov, Russo, and Chesnokov [25].

In [28] Zakharov considered the first nontrivial multicomponent reduction of the Benney system for a multilayered fluid, proved its integrability, and constructed a complete infinite set of conservation laws for this reduction. Zakharov's multilayer reduction has the following important property: at each point $x$ the horizontal components of the velocity within each layer are constant and distinct generally implying sliding between layers. This property does not allow one to apply Zakharov's reduction to the description of fluid flows with vertically varying continuous velocity profiles.

Stability of shear flows for the full Euler equations is a fundamental problem of fluid mechanics (see, e.g., [10]). The classical stability and instability criteria formulated in terms of growth of linear perturbations (Rayleigh, Fjortoft) are obtained only for flows between rigid walls. Some recent works use the generalized notion of stability as the well-posedness of time evolution, i.e., hyperbolicity (see $[7,8]$ ), but they also consider either flows between rigid walls, or use periodic boundary conditions in the vertical direction. However, the presence of a free surface can obviously change the flow stability criteria and, to our knowledge, stability of shallow water shear flows with a free surface has not been studied before. We note that, being an integro-differential system, the Benney equations cannot be directly classified in terms of the hyperbolicity property. A generalized theory of characteristics and the notion of hyperbolicity for integro-differential equations of the long wave theory was introduced by Teshukov [23, 24] and Liapidevskii and Teshukov [18]. They developed new mathematical tools for the qualitative study of integro-differential hyperbolic equations. Applying this technique, Chesnokov and Khe [5] revealed an analogue of Landau damping for the Benney equations.

The structure of the paper is as follows. In section 2, we present the necessary preliminaries on the hyperbolicity of the Benney equations in the sense of [23, 24]. We then show that, if the velocity profile over the vertical coordinate varies smoothly, then its monotonicity and convexity is sufficient for the stability of flows with a free surface. We also extend this result for the Fjortoft-like velocity profiles. Using the Vlasov-like formulation of the governing equations we reveal an analogy between the criteria for the stability of plasma waves and shear flows. In section 3 we derive the models describing flows with a piecewise linear (continuous or not) velocity profile. We then formulate sufficient conditions for the stability of such multilayer flows. The stability study is based on the verification of the hyperbolicity condition. We also reveal some important mathematical properties of the equations for multilayer flows (the existence of Riemann invariants and the Hamiltonian structure). It should be stressed that our approximation of the Benney equations for shear flows with piecewise constant vorticity is an important "upgrade" of the classical Zakharov reduction [28] as it admits a class of physically natural continuous velocity profiles. In section 4, we consider two-layer stratified flows with a piecewise linear velocity profile. We show that the generalization of previous results to the case of stratified flows is hardly possible. Nevertheless, we can state that the presence of vorticity has a stabilizing effect on the flow of stratified fluid. Finally, in section 5 we draw some conclusions from our study.

2. Benney equations and the hyperbolicity condition. Consider the twodimensional time-dependent flow of an inviscid homogeneous fluid under the action 
of gravity $g$. Let $z=0$ and $z=h(t, x)$ be the positions of the rigid bottom and free surface, respectively. The dimensionless Euler equations governing the motion are

$$
u_{t}+u u_{x}+w u_{z}+p_{x}=0, \quad \varepsilon^{2}\left(w_{t}+u w_{x}+w w_{z}\right)+p_{z}=-g, \quad u_{x}+w_{z}=0 .
$$

The boundary conditions are:

$$
\left.w\right|_{z=0}=0, \quad h_{t}+u h_{x}-\left.w\right|_{z=h}=0,\left.\quad p\right|_{z=h}=p_{0} .
$$

Here $u=V^{-1} u^{*}, w=L\left(V H_{0}\right)^{-1} w^{*}, p=\left(\rho V^{2}\right)^{-1} p^{*}, x=L^{-1} x^{*}, z=H_{0}^{-1} z^{*}$, $t=V L^{-1} t^{*}$, and $g=H_{0} V^{-2} g^{*}$ are dimensionless components of the velocity, pressure, Cartesian coordinates, time, and gravity, respectively; $u^{*}, w^{*}, p^{*}, x^{*}, z^{*}, t^{*}$, and $g^{*}$ are the corresponding dimensional variables. The parameters $L, H_{0}$, and $V$ denote the horizontal scale of the wave, the mean depth, and the characteristic velocity, respectively; the dimensionless parameter $\varepsilon=H_{0} / L$. The constants $\rho$ and $p_{0}$ are the density of the fluid and the dimensionless atmospheric pressure, respectively. The vorticity of a two-dimensional flow has only one nontrivial component $-\omega=$ $H_{0} V^{-1} \omega^{*}$, where $\omega=u_{z}-\varepsilon^{2} w_{x}$. It conserves along the trajectories

$$
\omega_{t}+u \omega_{x}+w \omega_{z}=0 .
$$

If the waves are long, i.e., the dimensionless parameter is small, $\varepsilon \ll 1$, and at leading order in $\varepsilon$ the pressure is hydrostatic so that $p=g(h-y)+p_{0}$, the problem (1), (2) reduces to the system of Benney equations [1]

$$
\begin{aligned}
& u_{t}+u u_{x}+w u_{z}+g h_{x}=0, \\
& h_{t}+\left(\int_{0}^{h} u d z\right)_{x}=0, \quad w=-\int_{0}^{z} u_{x}\left(t, x, z^{\prime}\right) d z^{\prime} .
\end{aligned}
$$

This system describes the propagation of nonlinear long waves in a shear flow with a free surface. From (4) one can deduce that the long-wave vorticity $\omega=u_{z}$ also satisfies (3). The classical shallow water theory corresponds to the irrotational motion $u=u(t, x), w=-z u_{x}$. The flow is described by the well-known nonlinear shallow water equations (Saint-Venant equations):

$$
u_{t}+u u_{x}+g h_{x}=0, \quad h_{t}+(u h)_{x}=0 .
$$

Generalized hyperbolicity conditions for the integro-differential equations (4) on a solution $u(t, x, z), h(t, x)$ with a monotonic velocity profile (e.g. $\left.u_{z}>0\right)$ are formulated in $[23,18,25]$ in terms of the characteristic function

$$
\chi(k)=1-g \int_{0}^{h} \frac{d z}{(u-k)^{2}}=1+g \int_{0}^{h} \frac{1}{u_{z}} \frac{\partial}{\partial z}\left(\frac{1}{u-k}\right) d z .
$$

More precisely, let us consider the limit values of $\chi(k)$ on the interval $\left[u_{b}, u_{s}\right]$ (here the subscripts $b$ and $s$ correspond to the values of $\chi(k)$ at $z=0$ and $z=h$, respectively) from the upper and lower complex half-planes:

$$
\chi^{ \pm}(u)=1+g\left(\frac{W_{s}}{u_{s}-u}-\frac{W_{b}}{u_{b}-u}-\int_{0}^{h} \frac{W_{z}^{\prime} d z^{\prime}}{u^{\prime}-u} \mp \pi i \frac{W_{z}}{u_{z}}\right) .
$$

Relations (6) are obtained from (5) by integration by parts and the application of the Sokhotski-Plemelj formulas. Here $W=1 / u_{z}, u^{\prime}=u\left(t, x, z^{\prime}\right), W^{\prime}=W\left(t, x, z^{\prime}\right)$. 


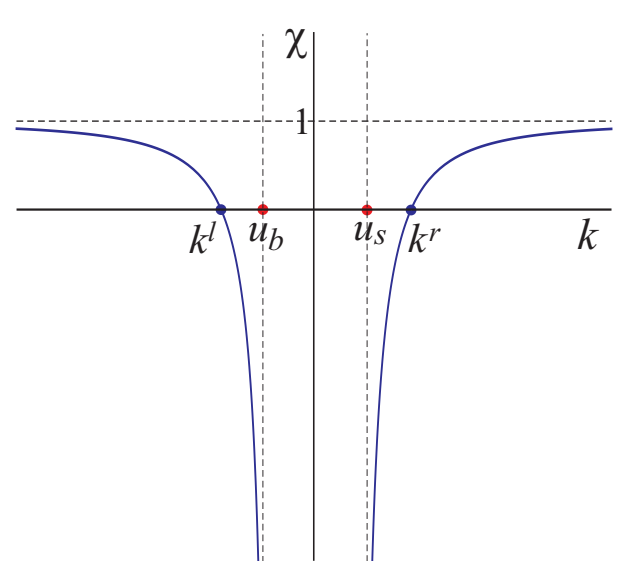

FIG. 1. A typical graph of the function $\chi(k), k \in\left(-\infty, u_{b}\right) \cup\left(\infty, u_{s}\right)$, for a monotonic $\left(u_{z}>0\right)$ velocity profile.

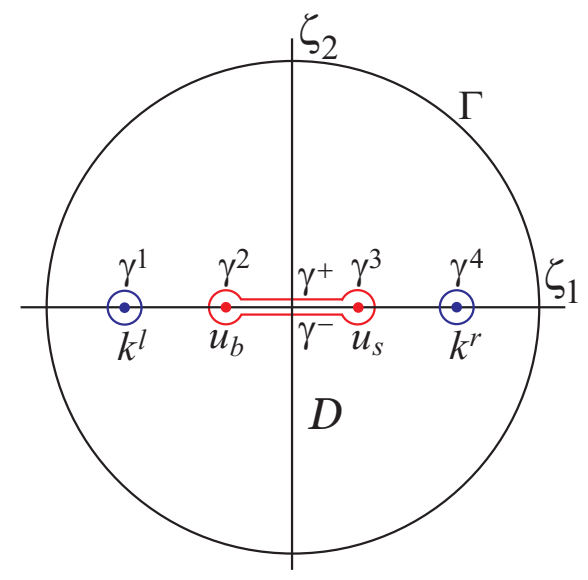

FIG. 2. Contour in the complex plane $\zeta$ used for the formulation of the hyperbolicity conditions.

Let the bounded function $W>0$ be differentiable with respect to $z$, and $W_{z}$ is Hölder continuous on the interval $z \in[0, h]$. Then the characteristic equation $\chi(k)=0$ has exactly two real roots $k=k^{l}<u_{b}$ and $k=k^{r}>u_{s}$ (see Figure 1). Indeed, $\chi(k) \rightarrow 1$ if $k \rightarrow \pm \infty ; \chi(k) \rightarrow-\infty$ if $k \rightarrow u_{b}-0$ or $k \rightarrow u_{s}+0 ; \chi^{\prime}(k)<0$ for $k<u_{b}$ and $\chi^{\prime}(k)<0$ for $k>u_{s}$. Equations (4) are hyperbolic (in the sense of $[23,24])$ if the following condition holds:

$$
\Delta \arg \frac{\chi^{+}(u)}{\chi^{-}(u)}=0, \quad \chi^{ \pm}(u) \neq 0 .
$$

The argument increment is calculated when $z$ changes from 0 to $h$ at fixed values of $t$ and $x$.

The condition (7) ensures, in particular, the absence of complex roots of the characteristic equation $\chi(k)=0$. Consider the domain $D$ (see Figure 2) in the plane of complex variable $\zeta=\zeta_{1}+i \zeta_{2}$, bounded by a circle $\Gamma$ of radius $R_{\varepsilon}$ with center at the origin of coordinates, circles $\gamma^{j}$ of radii $r_{\varepsilon}$ with centers at the points $k^{l}, k^{r}, u_{b}$, $u_{s}$, and segments $\gamma^{ \pm}$of the cut sides $\left(u_{b}, u_{s}\right)$. One supposes that $r_{\varepsilon} \rightarrow 0, R_{\varepsilon} \rightarrow \infty$ as $\varepsilon \rightarrow 0$. The increment in the argument of the function $\chi(\zeta)$ along the boundary of the domain $D$ normalized by $2 \pi$ is equal to the number of zeros of the function $\chi(\zeta)$ in this domain. Indeed, $\chi(\zeta)$ has no poles in the domain $D$. Moreover, $\chi(\zeta)$ has first-order zeros at the points $\zeta=k^{l}, \zeta=k^{r}$ and first-order poles at the points $\zeta=u_{b}$, $\zeta=u_{s}$. Thus, $\chi(\zeta)$ has no zeros in the domain $D$ if the increment of its argument along segments $\gamma^{ \pm}$is equal to zero.

Following $[23,18,25]$ we introduce the Riemann invariants

$$
R=u-g \int_{0}^{h} \frac{d z^{\prime}}{u^{\prime}-u}, \quad r^{i}=k^{i}-g \int_{0}^{h} \frac{d z}{u-k^{i}} \quad(i=l, r) .
$$

(Note that (3) is already in Riemann form with $\omega=u_{z}$ being the Riemann invariant). Here $k^{l}$ and $k^{r}$ are the roots of the characteristic equation $\chi(k)=0$. Let the functions $u(t, x, z)$ and $h(t, x)$ be a solution of (4), then the Riemann invariants satisfy the equations 


$$
\begin{aligned}
& R_{t}+u R_{x}+w R_{z}=0, \quad \omega_{t}+u \omega_{x}+w \omega_{z}=0, \\
& r_{t}^{l}+k^{l} r_{x}^{l}=0, \quad r_{t}^{r}+k^{r} r_{x}^{r}=0 .
\end{aligned}
$$

According to $[23,18]$ systems (4) and (9) are equivalent on smooth solutions if the hyperbolicity condition (7) holds.

Remark 2.1. Characteristic properties of (4) for flows with a nonmonotonic velocity profile were studied in [26]. Let the function $u(t, x, z)$ satisfy the following conditions:

$$
\begin{aligned}
& u_{z}>0 \quad \text { for } \quad z \in\left[0, z_{*}(t, x)\right), \quad u_{z}<0 \quad \text { for } \quad z \in\left(z_{*}(t, x), h(t, x)\right], \\
& u_{z z}\left(t, x, z_{*}\right) \neq 0, \quad u_{b}=u(t, x, 0)<u(t, x, h)
\end{aligned}
$$

i.e., $z=z_{*}$ is the maximum point for $u$ as a function of $z$. We define the complex function

$$
\chi_{1}(\zeta)=\left(\zeta-u_{*}\right)\left(1-g \int_{0}^{h} \frac{d z}{(u-\zeta)^{2}}\right)
$$

where $u_{*}=u\left(t, x, z_{*}\right)$. According to [26] the generalized hyperbolicity conditions for (4) for flows of class (10) are formulated as follows:

$$
\frac{1}{\pi} \Delta \arg \frac{\chi_{1}^{+}(u)}{\chi_{1}^{-}(u)}=-3, \quad \chi^{ \pm}(u) \neq 0 .
$$

Here $\chi_{1}^{ \pm}(u)$ are the limiting values of $\chi_{1}(\zeta)$ from the upper and lower complex halfplanes on the segment $\left[u_{b}, u_{*}\right]$. The argument increment is calculated when $u$ changes from $u_{b}$ to $u_{*}$.

Unfortunately, singularity of $1 / u_{z}$ at the point $z=z_{*}$ does not allow one to represent the function $\chi_{1}(\zeta)$ in the form of the Cauchy-type integral (as was done in (5) by integration by parts) and define the functions $\chi_{1}^{ \pm}(u)$. For this reason we restrict our consideration here to flows with monotonic velocity profiles.

2.1. Stability analysis. Let us study the stability of shear flows with a free surface. We say that the flow is stable if the corresponding system of equations is hyperbolic. In particular, we show that for smooth flows with a monotonic and convex velocity profile the hyperbolicity condition (7) is always fulfilled.

Let $u=U(z), U^{\prime}(z)>0$ (the variables $t$ and $x$ are fixed). In the verification of the hyperbolicity condition (7), it is convenient to use the functions

$$
\Psi^{ \pm}(U)=m(U) \chi^{ \pm}(U), \quad m(U)=\left(U_{1}-U\right)\left(U-U_{0}\right) \geq 0
$$

which have no poles at the boundary points $U_{0}=U(0)$ and $U_{1}=U(h)$. Here the complex functions $\chi^{ \pm}$are defined by (6). Following [18], in the plane $\left(Z_{1}, Z_{2}\right)$ we construct a closed contour $C$ consisting of the contours $C^{-}$and $C^{+}$. The contour $C^{-}$ is given parametrically by the equations

$$
Z_{1}=\operatorname{Re}\left\{\Psi^{-}(U)\right\}, \quad Z_{2}=\operatorname{Im}\left\{\Psi^{-}(U)\right\}
$$

for $U$ varying from $U_{0}$ to $U_{1}$. A contour $C^{+}$, which is symmetric to the contour $C^{-}$ with respect to the $Z_{1}$ axis, is given by the same equations but with the function $\Psi^{+}(U)$. The contour $C$ allows one to determine the argument increment of the functions $\Psi^{ \pm}(U)$ (or $\chi^{ \pm}(U)$ ) when $U$ changes from $U_{0}$ to $U_{1}$. According to the argument 
principle, if the point of $Z_{1}=0, Z_{2}=0$ lies in the domain bounded by the contour $C$, then the argument increment of $\Psi^{ \pm}$is not equal to zero. This means that condition (7) is violated and, consequently, the characteristic equation $\chi(k)=0$ has complex roots (the function $\chi(k)=0$ is given by (5)). Otherwise, if the point $Z_{1}=0, Z_{2}=0$ is not in the domain bounded by the contour $C$, condition (7) is satisfied and the governing equations (4) for the corresponding solution are hyperbolic.

Taking into account the identity $W_{z}=\left(1 / U^{\prime}\right)^{\prime}=-U^{\prime \prime} /\left(U^{\prime}\right)^{2}$ one obtains

$$
\begin{aligned}
\Psi^{ \pm}(U)= & m(U)\left(1+g \int_{0}^{h} \frac{U^{\prime \prime}(\xi) d \xi}{\left(U^{\prime}(\xi)\right)^{2}(U(\xi)-U(z))}\right) \\
& +g\left(\frac{U-U_{0}}{U_{1}^{\prime}}+\frac{U_{1}-U}{U_{0}^{\prime}}\right) \pm g \pi i \frac{m(U) U^{\prime \prime}}{\left(U^{\prime}\right)^{3}} .
\end{aligned}
$$

We will use these functions to prove the following main statements.

Lemma 2.2. Let $U^{\prime \prime}(z) \neq 0$, then the flow is stable (Rayleigh-like criterion).

Lemma 2.3. Let $U^{\prime \prime}<0$ for $z \in\left[0, z_{c}\right), U^{\prime \prime}\left(z_{c}\right)=0$, and $U^{\prime \prime}>0$ for $z \in\left(z_{c}, h\right]$. Then the flow is stable.

Lemma 2.4. Let $U^{\prime \prime}>0$ for $z \in\left[0, z_{c}\right), U^{\prime \prime}\left(z_{c}\right)=0$, and $U^{\prime \prime}<0$ for $z \in\left(z_{c}, h\right]$. Then the flow may be unstable.

We now present the proofs of these statements.

Proof of Lemma 2.2. If $U^{\prime \prime}(z) \neq 0$, then the functions $\operatorname{Im} \Psi^{ \pm}(U)$ have a constant sign in the interval $\left(U_{0}, U_{1}\right)$ because $U^{\prime}>0, m(U)>0$, and $U^{\prime \prime} \neq 0$. For $U=U_{0}$ and $U=U_{1}$ the imaginary part of $\Psi^{ \pm}(U)$ vanishes and the functions take the following values at these points:

$$
\Psi^{ \pm}\left(U_{0}\right)=g \frac{U_{1}-U_{0}}{U_{0}^{\prime}}>0, \quad \Psi^{ \pm}\left(U_{1}\right)=g \frac{U_{1}-U_{0}}{U_{1}^{\prime}}>0 .
$$

A typical velocity profile $u=U(z)$ and corresponding contour $C^{-}$are shown in Figure 3. This figure is obtained for the function

$$
U(z)=\frac{(z+1)^{-3 / 4}-1}{2^{-3 / 4}-1}, \quad z \in[0,1]
$$

with $g=1$ (in this case $U^{\prime \prime}<0$ ). The point $Z_{1}=0, Z_{2}=0$ is not in the domain bounded by the contour $C$. This means that the arguments of the complex functions $\Psi^{ \pm}(U)$ do not increase as $U$ is changed from $U_{0}$ to $U_{1}$ and, consequently, the hyperbolicity condition (7) is satisfied.

Proof of Lemma 2.3. This statement is a Fjortoft-like criterion which can also be written in the following form. Let

$$
\left(U(z)-U_{c}\right) U^{\prime \prime}(z) \geq 0, \quad z \in[0, h],
$$

then the flow is stable. Here $z=z_{c}$ is an inflection point at which $U^{\prime \prime}\left(z_{c}\right)=0$ and $U_{c}=U\left(z_{c}\right)$. By the definition of the functions $\Psi^{ \pm}$, the sign of $\operatorname{Im} \Psi^{ \pm}$coincides with the sign of $U^{\prime \prime}$. As before, at the boundary points $U_{0}$ and $U_{1}$ the functions $\Psi^{ \pm}$take the positive values. Therefore, the question of the satisfaction of the hyperbolicity 

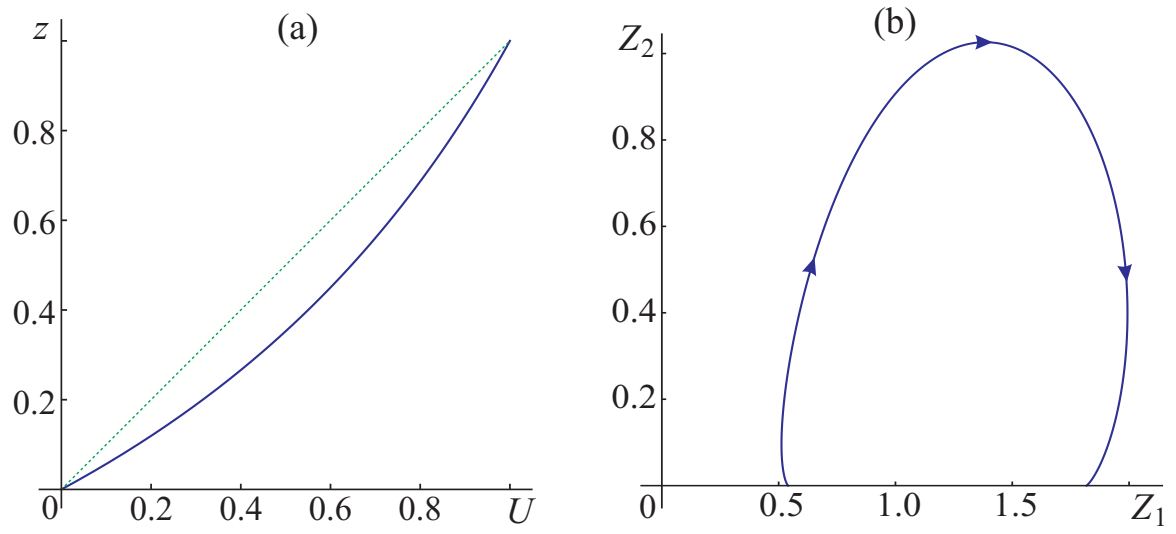

FIG. 3. An example of a monotonic convex velocity profile $u=U(z)$ (a) and the corresponding contour $C^{-}$in the complex plane $\left(Z_{1}, Z_{2}\right)$ (b) (the arrows indicate the direction of the path tracing).
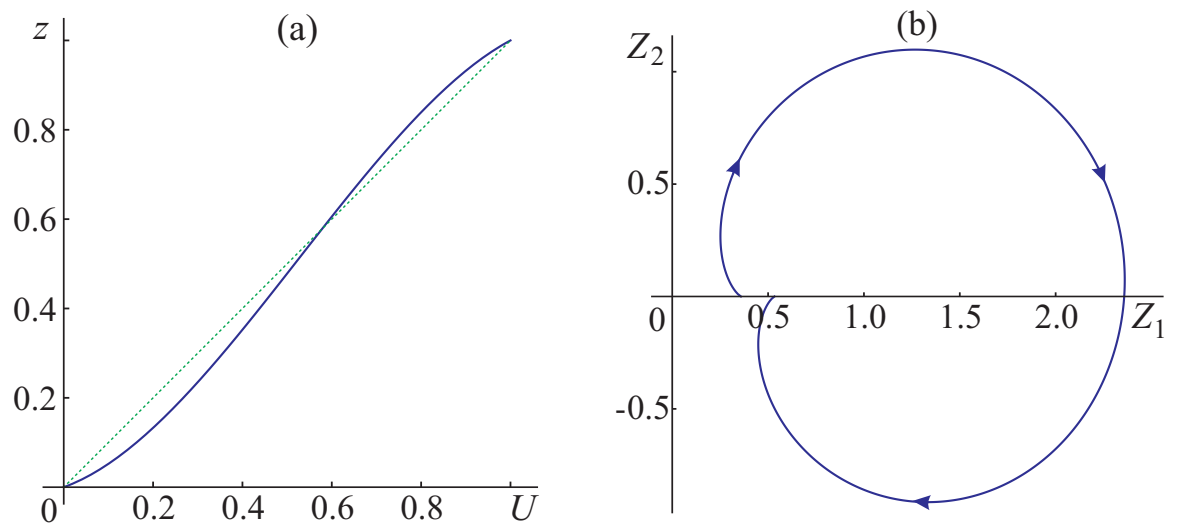

FIG. 4. An example of a monotonic velocity profile (solid line) which satisfies condition (12) (a) and the corresponding contour $C^{-}$in the complex plane $\left(Z_{1}, Z_{2}\right)$ (b).

condition (7) reduces to validating the inequality $\Psi^{ \pm}\left(U_{c}\right)>0$. When inequality (12) is satisfied, we have

$$
\Psi^{ \pm}\left(U_{c}\right)=g\left(\frac{U_{c}-U_{0}}{U_{1}^{\prime}}+\frac{U_{1}-U_{c}}{U_{0}^{\prime}}\right)+m\left(U_{c}\right)\left(1+g \int_{0}^{h} \frac{\left(U(z)-U_{c}\right) U^{\prime \prime}(z) d z}{\left(U^{\prime}(z)\right)^{2}\left(U(z)-U_{c}\right)^{2}}\right)>0
$$

since all the terms of the expression are positive.

A typical velocity profile satisfying condition (12) and the corresponding contour $C^{-}$are shown in Figure 4, which is obtained for the function

$$
z(U)=\left(\left(1-\frac{a_{1} U}{3}\right) \frac{a_{2} U}{2}+1+\frac{\left(a_{1}-3\right) a_{2}}{6}\right) U, \quad U \in[0,1]
$$

(here $\left.U_{c}=1 / a_{1}\right)$, with $a_{1}=1.9, a_{2}=3.5, g=1$.

Proof of Lemma 2.4. Although at the boundary points inequalities (11) are still satisfied and the imaginary part of the functions $\Psi^{ \pm}$changes sign once with a change in $U$ from $U_{0}$ to $U_{1}$, we cannot guarantee that $\Psi^{ \pm}\left(U_{c}\right)>0$. Indeed, let us consider the following class of velocity profiles 

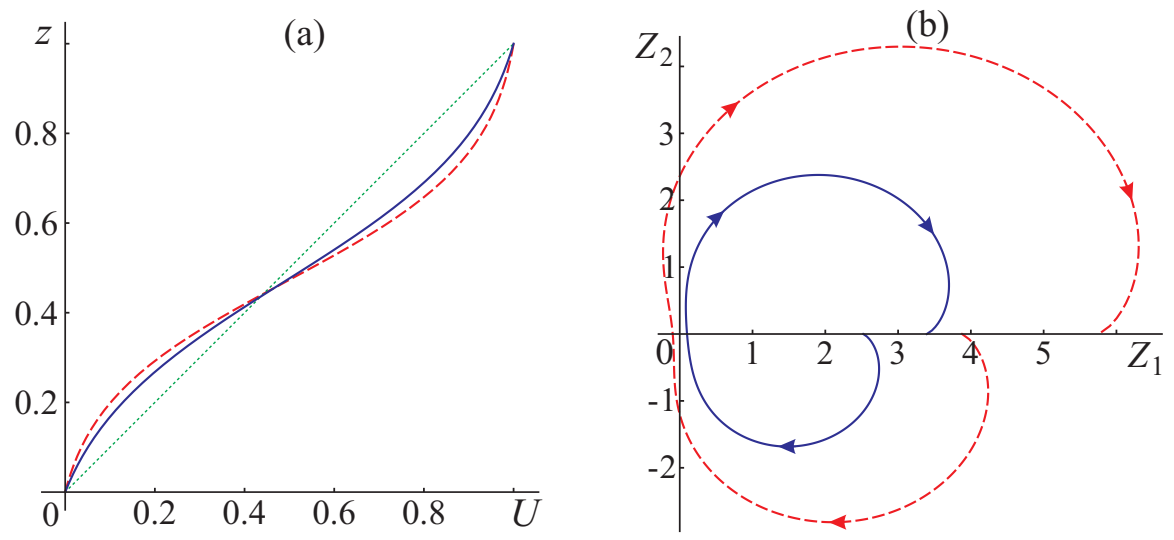

FIG. 5. Monotonic velocity profiles from class (13) (a) and corresponding contours $C^{-}$(b) obtained for $z_{c}=0.47, a=2.8$ (solid lines), and $a=3.5$ (dashed lines).

$$
U(z)=\frac{\tanh \left(\left(z-z_{c}\right) a\right)+\tanh \left(a z_{c}\right)}{\tanh \left(\left(1-z_{c}\right) a\right)+\tanh \left(a z_{c}\right)}, \quad z \in[0,1]
$$

which corresponds to the considered case. Here $z_{c}$ is inflection point and $U^{\prime \prime \prime}\left(z_{c}\right)<0$. The parameter $a$ affects the rate of change of the function $U(z)$ near the inflection point: the velocity profile tends to a discontinuous piecewise constant function as $a \rightarrow \infty$.

A velocity profile belonging to class (13) and the corresponding contour $C^{-}$are shown in Figure 5 (solid lines) for $z_{c}=0.47, a=2.8$, and $g=1$. As we can see from Figure $5(\mathrm{~b})$ the point of $Z_{1}=0, Z_{2}=0$ is not in the domain bounded by the contour $C$. Consequently, the hyperbolicity condition (7) is fulfilled. Dashed lines in Figure 5 are obtained for $a=3.5$ (the others parameters are the same). In this case the point of $Z_{1}=0, Z_{2}=0$ belongs to the domain bounded by the contour $C$ and the hyperbolicity condition (7) is violated.

We have proved that the classical stability criteria for shear flows of ideal fluid [10] correspond to the hyperbolicity condition (7) of the governing equations (4). Thus the Rayleigh-Fjortoft criteria (for flows with monotonic velocity profile) provide the hyperbolicity of the flow with a free surface, i.e., it is a sufficient condition of the stability for vortex shallow water flows. The same correspondence between the classical stability criteria and the hyperbolicity condition for the integro-differential equations of the long-wave theory for the flow between rigid walls was established in [15].

Let us also remark that the convexity of the velocity profile is not sufficient for the stability, if the dispersive terms are added. For the Serre-Green-Naghdi-type equations the stability criterion was established in [13] where the additional (with respect to the convexity and monotonicity conditions) inequalities were added to guarantee the flow stability even for the case of the flow between walls.

2.2. Vlasov-like formulation. Governing equations (4) admit a kinetic formulation [28] in the case of flows with nonzero vorticity (we choose $u_{z}>0$ as before). Following [25] we make a change of variables to new independent $t, x, u$ and dependent $W=1 / u_{z}, u_{b}(t, x)=u(t, x, 0), u_{s}(t, x)=u(t, x, h)$ ones. For the unknown functions $W(t, x, u), u_{b}(t, x)$, and $u_{s}(t, x)$ we obtain a closed integro-differential model 


$$
\begin{aligned}
& W_{t}+u W_{x}-g h_{x} W_{u}=0, \quad h=\int_{u_{b}}^{u_{s}} W d u, \\
& u_{b t}+u_{b} u_{b x}+g h_{x}=0, \quad u_{s t}+u_{s} u_{s x}+g h_{x}=0,
\end{aligned}
$$

which is analogous to the Vlasov kinetic equation. The variable $W$, which is inversely proportional to the long-wave vorticity, acts as a distribution function.

Indeed, due to the identities

$$
d u=u_{t} d t+u_{x} d x+u_{z} d z, \quad d z=z_{t} d t+z_{x} d x+z_{u} d u
$$

we have

$$
u_{t}=-z_{t} u_{z}, \quad u_{x}=-z_{x} u_{z}, \quad u_{z} z_{u}=1
$$

Substituting relations (15) into the first equation of (4) one obtains

$$
z_{t}+u z_{x}-w-g h_{x} W=0,
$$

where $W=z_{u}$. Further, we differentiate the above equation with respect to $u$ and take into account that $z_{x}-w_{u}=0$ (this formula is a direct consequence of the definition of $w$ following from (4)). As a result, we obtain the first equation of system (14).

As a consequence of (4) we obtain

$$
u_{j t}+u_{j} u_{j x}+g h_{x}=0 \quad(j=b, s) .
$$

While the equation for the velocity $u_{b}$ at the bottom $y=0$ is straightforward, the second equation for velocity $u_{s}=u$ at the free surface $z=h$ is less obvious. It can be obtained as follows:

$$
\begin{aligned}
u_{s t}+u_{s} u_{s x}+g h_{x} & =u_{t}+u u_{x}+\left.u_{z}\left(h_{t}+u h_{x}\right)\right|_{z=h}+g h_{x} \\
& =u_{t}+u u_{x}+w u_{z}+\left.g h_{x}\right|_{z=h}=0 .
\end{aligned}
$$

The second equation in (4) reads

$$
\left(\int_{u_{b}}^{u_{s}} W d u\right)_{t}+\left(\int_{u_{b}}^{u_{s}} u W d u\right)_{x}=0 .
$$

It is easy to verify that this equation is fulfilled by virtue of (14).

Remark 2.5. Kinetic formulation (14) of the Benney equations (4) allows one to reveal an analogy between the stability criteria for plasma waves and shear flows. It is known [21] that any solution of the one-dimensional linearized Vlasov equation is stable if it is defined by a distribution function with a single maximum. Obviously, the functions $W=W(u)$ with one maximum obey the inequality

$$
\left(u_{c}-u\right) W^{\prime}(u) \geq 0
$$

where $u=u_{c}$ is the extremum (maximum) point. Since the velocity profile $u=U(z)$ is related to the distribution function $W=W(u)$ as $u_{z}=1 / W$ and, consequently, $W_{u}=-u_{z z} / u_{z}^{3}$, we obtain the Fjortoft stability criterion (12). 
(a)

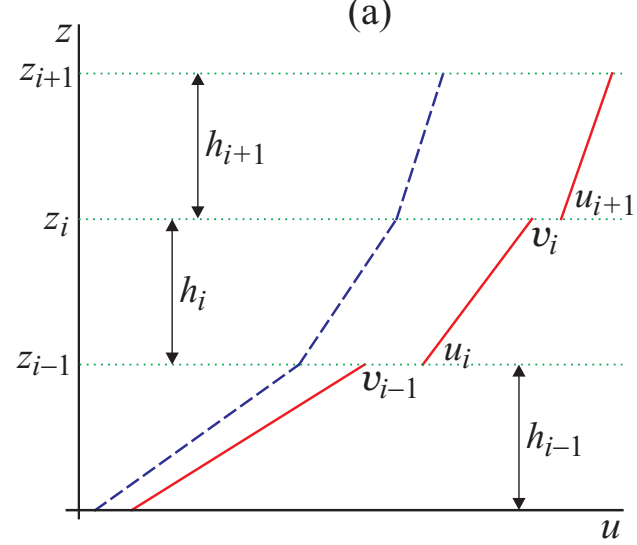

(b)

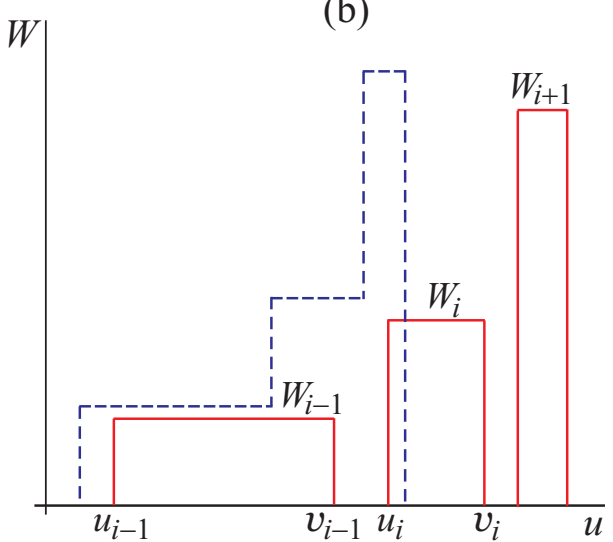

FIG. 6. An example of a piecewise linear velocity profile (the variables $t$ and $x$ are fixed) (a) and the corresponding "kinetic" ansatz (b); solid lines-general case, dashed lines-flow with piecewise constant vorticity and a continuous velocity profile.

3. Class of piecewise linear velocity profiles: Governing equations. Let us consider the class of flows with a piecewise linear velocity profile (see Figure 6)

$$
u=\omega_{i}\left(z-z_{i-1}\right)+u_{i}, \quad z \in\left(z_{i-1}, z_{i}\right), \quad i=1, \ldots, N,
$$

and introduce the following notations. Each $i$ th layer is characterized by the velocity $u_{i}(t, x)$ at the lower boundary and the depth $h_{i}(t, x)=z_{i}-z_{i-1}$, as well as by the constant vorticity $\omega_{i}$. We also introduce velocity $v_{i}(t, x)=\omega_{i} h_{i}+u_{i}$ at the upper boundary of the layer and the average velocity $\bar{u}_{i}(t, x)=\left(v_{i}+u_{i}\right) / 2=u_{i}+\omega_{i} h_{i} / 2$. It is obvious that $z_{0}=0, z_{N}=h=h_{1}+\cdots+h_{N}$. We also note that due to the definition of $v_{i}$ we have

$$
v_{i t}+v_{i} v_{i x}+g h_{x}=u_{i t}+u_{i} u_{i x}+g h_{x}+\omega_{i}\left(h_{i t}+\left(\bar{u}_{i} h_{i}\right)_{x}\right), \quad i=1, \ldots, N .
$$

At the upper and lower boundaries of each layer the following kinematic conditions should be satisfied:

$$
\frac{\partial z_{i}}{\partial t}+v_{i} \frac{\partial z_{i}}{\partial x}=w_{i}^{+}, \quad \frac{\partial z_{i-1}}{\partial t}+u_{i} \frac{\partial z_{i-1}}{\partial x}=w_{i}^{-}, \quad i=1, \ldots, N .
$$

Here $w_{i}^{+}$and $w_{i}^{-}$are the values of the vertical velocity $w$ at $z=z_{i}-0$ and $z=z_{i-1}+0$, respectively. Taking into account representation (16), equation $u_{x}+w_{z}=0$, and kinematic condition (18) at $z=z_{i}-0$ one can express velocity $w$ in the form

$$
w=-\left(z-z_{i-1}\right)\left(\frac{\partial u_{i}}{\partial x}-\omega_{i} \frac{\partial z_{i-1}}{\partial x}\right)+\frac{\partial z_{i-1}}{\partial t}+u_{i} \frac{\partial z_{i-1}}{\partial x}, \quad z \in\left(z_{i-1}, z_{i}\right) .
$$

Let us calculate the difference $w_{i}^{+}-w_{i}^{-}$using (18) and formula (19). As a result, we obtain

$$
h_{i t}+\left(\bar{u}_{i} h_{i}\right)_{x}=0, \quad i=1, \ldots, N .
$$

Substitution of the velocities $u$ and $w$ given by formulas (16), (19) into the first equation of (4) yields

$$
u_{i t}+u_{i} u_{i x}+g h_{x}=0, \quad i=1, \ldots, N .
$$

Copyright (c) by SIAM. Unauthorized reproduction of this article is prohibited. 
Equations (20) and (21) form a closed system for $2 N$ unknown functions $h_{i}$ and $u_{i}$. In view of (17) we can formulate the system governing flows from the class (16) in terms of $h_{i}$ and $v_{i}$. If all $\omega_{i} \neq 0$, then one can also use the variables $u_{i}$ and $v_{i}$.

Remark 3.1. In terms of the Vlasov-like formulation (14) the class of solutions (16) corresponds to a piecewise constant distribution function $W$ in the form [6]

$$
W(t, x, u)=\sum_{i=1}^{N}\left(\theta\left(u-u_{i}(t, x)\right)-\theta\left(u-v_{i}(t, x)\right)\right) W_{i},
$$

where $\theta$ is the Heaviside step function, and $W_{i}=1 / \omega_{i}$ are positive constants. The functions $u_{i}$ and $v_{i}$ are ordered in such a way that $u_{i+1}>v_{i}$ (see Figure 6 ). Substitution of (22) into the first equation in (14) yields

$$
\sum_{i=1}^{N}\left(\left(v_{i t}+v_{i} v_{i x}+g h_{x}\right) \delta\left(u-v_{i}\right)-\left(u_{i t}+u_{i} u_{i x}+g h_{x}\right) \delta\left(u-u_{i}\right)\right) W_{i}=0,
$$

where $\delta$ is the Dirac delta function. Thus, we obtain the following system of $2 N$ PDEs

$$
\begin{aligned}
& v_{i t}+v_{i} v_{i x}+g h_{x}=0, \quad u_{i t}+u_{i} u_{i x}+g h_{x}=0, \quad i=1, \ldots, N, \\
& h=\sum_{j=1}^{N}\left(v_{j}-u_{j}\right) / \omega_{j},
\end{aligned}
$$

describing the piecewise constant ansatz (22) of the Vlasov-like model (14), which corresponds to a free surface shear flow with a piecewise linear velocity profile. Note that $u_{b}=u_{1}$ and $u_{s}=v_{N}$ so the second and third equations in (14) are already included in (23). Obviously, that (23) is equivalent to (20), (21) if $\omega_{i} \neq 0$. System (23) represents the so-called waterbag reduction of the Benney equations. This type of reduction of kinetic equations play important role in plasma physics [9]. We note that a system equivalent to (23) appears in [2] as a formal reduction of the dispersionless Kadomtsev-Petviashvili equation, outside any connection with vortical flows.

As was mentioned above, the shallow water equations for shear flows (4) can be rewritten in terms of the Riemann invariants (9) if the hyperbolicity condition (7) is satisfied. In particular, for a piecewise linear velocity profile the Riemann invariants defined by formulas (8) (see also [2]) are

$$
r^{i}=k^{i}-\sum_{j=1}^{N} \frac{g}{\omega_{j}} \ln \left|\frac{v_{j}-k^{i}}{u_{j}-k^{i}}\right|,
$$

where $k^{i}(t, x)$ are zeros of the characteristic function (5), i.e., the roots of the equation

$$
1-g \sum_{i=1}^{N} \frac{1}{\omega_{i}}\left(\frac{1}{v_{i}-k}-\frac{1}{u_{i}-k}\right)=0 .
$$

If system (23) is hyperbolic, it can be written in the form

$$
r_{t}^{i}+k^{i} r_{x}^{i}=0, \quad i=1, \ldots, 2 N
$$

Representation (26), in particular, allows one to construct solutions in the class of simple waves. The $m$ th family $(m=1, \ldots, 2 N)$ of simple waves satisfies the relations $r^{i}\left(u_{1}, \ldots, u_{N}, v_{1}, \ldots, v_{N}\right)=r_{0}^{i}=$ const $, \quad i \neq m, \quad k^{m}\left(u_{1}, \ldots, u_{N}, v_{1}, \ldots, v_{N}\right)=k(t, x)$, where $k(t, x)$ is a solution of the Hopf equation $k_{t}+k k_{x}=0$. 
In some cases it is convenient to rewrite system $(20),(21)$ in the form

$$
h_{i t}+\left(\bar{u}_{i} h_{i}\right)_{x}=0, \quad \bar{u}_{i t}+\left(\frac{\bar{u}_{i}^{2}}{2}+\frac{\omega_{i}^{2} h_{i}^{2}}{8}+g \sum_{j=1}^{N} h_{j}\right)_{x}=0, \quad i=1, \ldots, N .
$$

One can see that in the limit of zero vorticity, $\omega_{i} \rightarrow 0, i=1, \ldots, N$, system $(27)$ yields the Zakharov reduction of the Benney equations [28]. Importantly, as we will show in the next section, the presence in (27) of the terms related to vorticity enables one to describe multilayer flows with physically relevant, continuous, velocity profiles, not captured by the Zakharov reduction.

It should be noted that system (27) admits a canonical Hamiltonian formulation

$$
\frac{\partial h_{i}}{\partial t}=-\frac{\partial}{\partial x}\left(\frac{\partial H}{\partial \bar{u}_{i}}\right), \quad \frac{\partial \bar{u}_{i}}{\partial t}=-\frac{\partial}{\partial x}\left(\frac{\partial H}{\partial h_{i}}\right), \quad i=1, \ldots, N,
$$

where the Hamiltonian is

$$
H=\frac{1}{2} \sum_{j=1}^{N} \bar{u}_{j}^{2} h_{j}+\frac{g}{2}\left(\sum_{j=1}^{N} h_{j}\right)^{2}+\frac{1}{24} \sum_{j=1}^{N} \omega_{j}^{2} h_{j}^{3} .
$$

Equations (27) obviously admit the energy and momentum conservation laws, with the densities $H$ defined above and $P$ defined below:

$$
P=\sum_{j=1}^{N} \bar{u}_{j} h_{j}
$$

Apart from the Hamiltonian structure, the quasi-linear system (27) has a number of remarkable properties including the availability of infinitely many conservation laws and integrability. However, in this paper we focus only on the stability study leaving other aspects related to the mathematical properties of (27) for a separate publication.

3.1. Class of piecewise linear continuous velocity profiles: Hyperbolicity study. From system (23) one can derive that the variables $s_{i}=u_{i+1}-v_{i}$ satisfy the equations

$$
s_{i t}+\left(\frac{u_{i+1}+v_{i}}{2} s_{i}\right)_{x}=0 .
$$

Obviously, if $\left.s_{i}\right|_{t=0}=0$, then $s_{i}=0$ for all $t>0$. This follows from the uniqueness of the solution of the Cauchy problem for the above system. Thus, for a homogeneous fluid, if the initial velocity profile is continuous, it will stay continuous for all time. For the density-stratified fluid this statement is not valid.

Sliding between the layers is unusual for homogeneous fluids. Therefore, from the physical point of view it is more natural to consider flows with a continuous velocity profile. This naturally leads to an admissible, piecewise constant long-wave vorticity $\omega=-u_{z}$. Indeed, in view of the Benney system (4), the equation for vorticity can be rewritten as follows:

$$
\omega_{t}+\nabla \cdot(\omega \mathbf{u})=0
$$

where $\mathbf{u}=(u, w)$ is the velocity vector. Let $\Sigma(t) \subset \mathbb{R}^{2}(x, z)$ be a surface where the function $\omega$ has a discontinuity, and $\mathbf{n}$ is the unit normal vector to $\Sigma$. From the above equation we obtain the Rankine-Hugoniot condition

$$
\left[\left(u_{n}-V_{n}\right) \omega\right]=0 .
$$

Copyright $@$ by SIAM. Unauthorized reproduction of this article is prohibited. 
Here $u_{n}=\mathbf{u} \cdot \mathbf{n}$ is the velocity in the normal direction to $\Sigma$ and $V_{n}$ is the velocity of $\Sigma$ in the same direction, $[f]$ is the difference in the values of $f$ at the two sides of $\Sigma$. If $\Sigma$ is a surface of contact discontinuity, i.e., if $u_{n}=V_{n}$, then the Rankine-Hugoniot relations are satisfied for any discontinuity of the vorticity. Hence piecewise constant vorticity profiles are weak solutions of the Benney equations.

$N$-layer flows of homogeneous fluid with a piecewise constant vorticity and a continuous velocity profile are defined by (16), where $u_{i}=v_{i-1}$. In this case system (20), (21) takes the form

$$
\begin{aligned}
& h_{i t}+\left(\bar{u}_{i} h_{i}\right)_{x}=0, \quad i=1, \ldots, N, \\
& v_{0 t}+v_{0} v_{0 x}+g h_{x}=0, \quad h=\sum_{i=1}^{N} h_{i},
\end{aligned}
$$

and consists of $N+1$ equations for the depths $h_{i}$ and for the velocity $v_{0}$ at the bottom $z=0$. Here

$$
\bar{u}_{i}=v_{i}-\frac{\omega_{i} h_{i}}{2}, \quad v_{i}=v_{0}+\sum_{j=1}^{i} \omega_{j} h_{j} .
$$

The Riemann invariants for (30) are obtained from the general formulas (24) by the reduction $u_{j}=v_{j-1}$. We stress that system (30), (31) represents an integrable multilayer approximation of the Benney equations, which, unlike the Zakharov reduction [28], describe flows with continuous velocity profiles. As a matter of fact, the presence of the admissible piecewise constant vorticity plays the crucial role in our construction.

Now, using the generalized theory of characteristics $[23,18]$ introduced before, we formulate sufficient conditions for hyperbolicity of (30). System (30) can be written in the form

$$
\mathbf{u}_{t}+A(\mathbf{u}) \mathbf{u}_{x}=0
$$

where $\mathbf{u}=\left(h_{1}, \ldots, h_{N}, v_{0}\right)^{\mathrm{T}}$ is the unknown vector and $A(\mathbf{u})$ is the corresponding matrix. To find the eigenvalues of $A(\mathbf{u})$, one has to solve the equation

$$
D(k)=\operatorname{det}(A-k I)=0 .
$$

It is convenient to use the characteristic function $\chi(k)(5)$. We introduce the separate notation $\bar{\chi}(k)$ for this function evaluated on the piecewiselinear velocity profile (16) with the additional requirement of continuity $u_{i}=v_{i-1}$. Then, on using the second formula in (31), we obtain

$$
\begin{aligned}
\bar{\chi}(k) & =1+g \sum_{i=1}^{N} \frac{1}{\omega_{i}}\left(\frac{1}{v_{i}-k}-\frac{1}{v_{i-1}-k}\right)=1-\sum_{i=1}^{N} \frac{g h_{i}}{\left(v_{i}-k\right)\left(v_{i-1}-k\right)} \\
& =1-\frac{g}{\omega_{1}} \frac{1}{v_{0}-k}+\frac{g}{\omega_{N}} \frac{1}{v_{N}-k}-g \sum_{i=1}^{N-1}\left(\frac{1}{\omega_{i+1}}-\frac{1}{\omega_{i}}\right) \frac{1}{v_{i}-k} .
\end{aligned}
$$

The roots $k=k^{i}$ of the equation $\bar{\chi}(k)=0$ are the characteristic velocities of system (30), and the following relation between the polynomial $D(k)$ and $\bar{\chi}(k)$ holds:

$$
D(k)=\bar{\chi}(k) \prod_{j=0}^{N}\left(v_{j}-k\right) .
$$




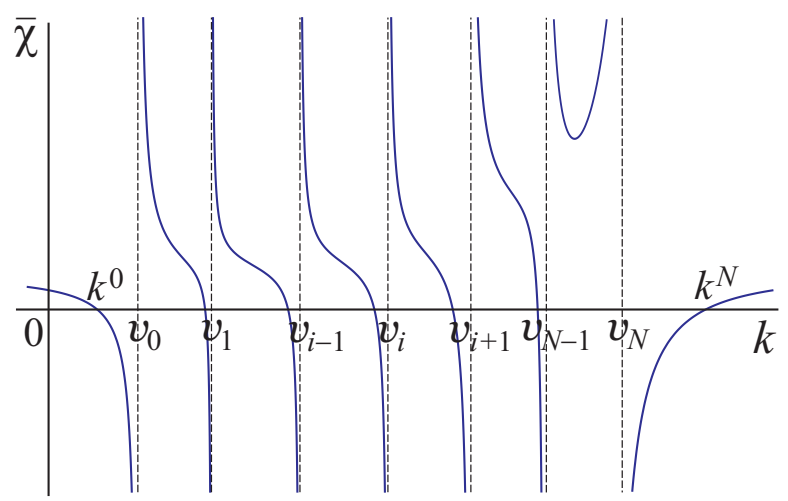

FIG. 7. A typical graph of the function $\bar{\chi}(k)$ for the case $\omega_{1}>\omega_{2}>\cdots>\omega_{N}>0$.

The derivative of the function $\bar{\chi}(k)$ is

$$
\bar{\chi}^{\prime}(k)=g \sum_{i=1}^{N} \frac{1}{\omega_{i}}\left(\frac{1}{\left(v_{i}-k\right)^{2}}-\frac{1}{\left(v_{i-1}-k\right)^{2}}\right) .
$$

LEMMA 3.2. Let all constant vorticities $\omega_{i}$ be ordered

$$
\omega_{N}<\omega_{N-1}<\cdots<\omega_{1} \quad \text { or } \quad \omega_{N}>\omega_{N-1}>\cdots>\omega_{1} .
$$

Then system (30) is hyperbolic.

Proof. Let us suppose that all $\omega_{i}$ have the same sign $\omega_{i}>0$. We prove the lemma statement for the case $\omega_{1}>\cdots>\omega_{N}$ (the other cases are treated similarly). In the intervals $k \in\left(-\infty, v_{0}\right)$ and $k \in\left(v_{N}, \infty\right)$ the equation $\bar{\chi}(k)=0$ has exactly two real roots $k^{0}<v_{0}$ and $k^{N}>v_{N}$ (see Figure 7). Indeed, $\bar{\chi}(k) \rightarrow 1$ if $k \rightarrow \pm \infty, \bar{\chi}(k) \rightarrow-\infty$ if $k \rightarrow v_{0}-0$, and $\bar{\chi}(k) \rightarrow \infty$ if $k \rightarrow v_{N}+0$. Moreover, $\bar{\chi}(k)^{\prime}<0$ if $k \in\left(-\infty, v_{0}\right)$, and $\bar{\chi}(k)^{\prime}>0$ if $k \in\left(v_{N}, \infty\right)$; see (35). The function $\bar{\chi}(k)$ is continuous on the intervals $k \in\left(v_{i}, v_{i+1}\right)$ and has the following limiting values

$$
\lim _{k \rightarrow v_{i}-0} \bar{\chi}(k)=-\infty, \quad \lim _{k \rightarrow v_{i}+0} \bar{\chi}(k)=\infty, \quad i=0, \ldots, N-1 .
$$

Thus, function $\bar{\chi}(k)$ changes sign in the intervals $k \in\left(v_{i-1}, v_{i}\right), i=1, \ldots, N-1$ and the equation $\bar{\chi}(k)=0$ has (at least) one root $k=k^{i}$ on each of these intervals (see Figure 7). We show that the function $\bar{\chi}(k)$ has at least $N+1$ zeros $k=k^{i}, k^{i} \neq v_{j}$. According to definition (33), $D(k)$ is a polynomial of order $N+1$ and, consequently, has $N+1$ roots. Taking into account relation (34) one can conclude that the function $\bar{\chi}(k)$ has exactly $N+1$ zeros $k=k^{i} \neq v_{j}$.

Let the vorticities $\omega_{i}$ be ordered and change sign such that

$$
\omega_{1}>\cdots>\omega_{j}>0>\omega_{j+1}>\cdots>\omega_{N}
$$

Since $\omega_{i+1}^{-1}-\omega_{i}^{-1}>0$ for all $i$ except for $i=j$, the function $\bar{\chi}(k)$ has the following limiting values,

$$
\lim _{k \rightarrow v_{i}-0} \bar{\chi}(k)=-\infty, \quad \lim _{k \rightarrow v_{i}+0} \bar{\chi}(k)=\infty, \quad i=0, \ldots, j-1, j+1, \ldots, N .
$$

Copyright $@$ by SIAM. Unauthorized reproduction of this article is prohibited. 
Suppose that all the characteristic velocities $v_{k}$ are pairwise distinct, i.e., $v_{i} \neq v_{m}$ for $i \neq m$ and thus can be ordered. To this end, instead of $v_{i}$ we introduce the variables $q_{i}$ $\left(q_{l}=v_{m}\right)$ which are ordered such that $q_{0}<q_{1}<\cdots<q_{N}=v_{j}$. In this case Figure 7 (where $q_{i}$ stand for $v_{i}$ ) also represents a typical graph of the function $\bar{\chi}(k)$ having $N+1$ real roots $k^{i} \neq v_{m}$. We also note, that if $v_{l}=v_{m}(l \leq j, m>j)$, then $k=v_{l}$ is a root of the equation $D(k)=0$. Hence, the cases of coinciding velocities $\left(v_{l}=v_{m}\right)$ and zero vorticity $\left(\omega_{j}=0\right)$ are also taken into consideration.

Thus, we proved that conditions (36) provide the existence of $N+1$ different characteristic roots $k=k^{i}$ of (33). This means that system (30) is hyperbolic.

Inequalities (36) imply that system (30) is strictly hyperbolic and hence the flow is stable in the sense of well-posedness of time evolution; see $[7,8]$. This sufficient condition (36) is reminiscent of the famous Rayleigh stability criterion about the shear flow stability between rigid walls: if the velocity profile is convex, the flow is stable. It weakens the criterion of stability proven in subsection 2.1 for the case of piecewise linear velocity profiles.

Remark 3.3. Equations (30) for two-layer flows $(N=2)$ are always hyperbolic because the two-layer velocity profile is always convex. Indeed, equation $\bar{\chi}(k)=0$ has two real roots $k^{l}<\min v_{j}$ and $k^{r}>\max v_{j}(j=0,1,2)$. Therefore, polynomial $D(k)$ has three real zeroes.

Remark 3.4. For multilayer flows the violation of conditions (36) may lead to the loss of hyperbolicity of (30). Let us consider the following example of a threelayer flow $(N=3)$ with unordered positive vorticities $\left(\omega_{1}<\omega_{2}, \omega_{3}<\omega_{2}\right)$. These parameters correspond to the piecewise linear approximation of a smooth nonconvex velocity profile of type (13) (see Figure 5) when a Fjortoft-like criterion (12) cannot be applied. We choose $g=1, h_{1}=h_{3}=1, h_{2}=\alpha \geq 0$, and $\omega_{1}=1 / 2, \omega_{2}=1, \omega_{3}=1 / 4$. It is easy to verify that there are four real roots of the characteristic equation $\bar{\chi}(k)=0$ if $\alpha>\alpha^{*} \approx 0.885$ or $0 \leq \alpha<\alpha_{*} \approx 0.031$. For $\alpha \in\left(\alpha_{*}, \alpha^{*}\right)$ there are only two real roots of the equation. Hence, system (30) is not hyperbolic in this case.

Let us choose positive constants $\omega_{i}>0$ such that $\omega_{1}>\omega_{2}, \omega_{3}>\omega_{2}$. It corresponds to three-layer $(N=3)$ piecewise linear approximation of the Fjortoft-like velocity profile (see Figure 4). In this case system (30) is always hyperbolic, because equation $\bar{\chi}(k)=0$ has four real roots: $k^{0}<v_{0}, k^{1} \in\left(v_{0}, v_{1}\right), k^{2} \in\left(v_{2}, v_{3}\right)$, and $k^{3}>v_{3}$. Indeed, $\omega_{2}^{-1}-\omega_{1}^{-1}>0$ and $\omega_{3}^{-1}-\omega_{2}^{-1}<0$. Hence, $\bar{\chi}(k) \rightarrow+\infty$ as $k \rightarrow v_{0}+0$ and $k \rightarrow v_{3}-0 ; \bar{\chi}(k) \rightarrow-\infty$ as $k \rightarrow v_{1}-0$ and $k \rightarrow v_{2}+0$. This means that there are roots $k^{1}$ and $k^{2}$ on the intervals $\left(v_{0}, v_{1}\right)$ and $\left(v_{2}, v_{3}\right)$, respectively.

4. Two-layer stratified flow with a piecewise constant vorticity. The generalization of the above results to the case of multilayer stratified flows is less obvious. Indeed, the fact that the densities in each layer are different implies that even if the sliding at the fluid interfaces is absent initially, it can emerge during the evolution. So, a continuous velocity profile does not exist in this case. The remarkable fact of the existence of Riemann invariants for homogeneous multilayer systems is also absent for stratified $N$-layer flows. Indeed, our calculation of the Haantjes tensor (see Appendix A) shows that it vanishes identically only in the case of homogeneous fluids.

We present here the hyperbolicity analysis for two-layer stratified flows. A general two-layer system is composed of two immiscible fluids of different constant densities $\rho_{1}$ and $\rho_{2}$ confined between the upper free surface and the lower rigid boundary. 


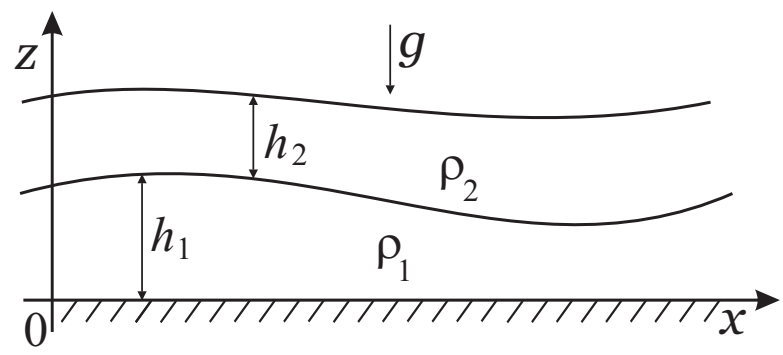

FIG. 8. Schematic of the two-layer stratified flow.

The shear flow in the long-wave approximation is governed by the equations [4]

$$
\begin{aligned}
& u_{t}^{1}+u^{1} u_{x}^{1}+w^{1} u_{z}^{1}+g h_{1 x}+g \rho h_{2 x}=0, \quad h_{1 t}+\left(\int_{0}^{h_{1}} u^{1} d z\right)_{x}=0, \\
& u_{t}^{2}+u^{2} u_{x}^{2}+w^{2} u_{z}^{2}+g h_{1 x}+g h_{2 x}=0, \quad h_{2 t}+\left(\int_{h_{1}}^{h_{1}+h_{2}} u^{2} d z\right)_{x}^{z}=0, \\
& w^{1}=-\int_{0}^{z} u_{x}^{1}\left(t, x, z^{\prime}\right) d z^{\prime}, \quad w^{2}=-\int_{h_{1}}^{z} u_{x}^{2}\left(t, x, z^{\prime}\right) d z^{\prime}+h_{1 t}+u^{2}\left(t, x, h_{1}\right) h_{1 x} .
\end{aligned}
$$

Here the variables $u^{i}(t, x, z), w^{i}(t, x, z)$ and $h_{i}(t, x)$ are the velocity components and the layer depths; $g$ is the gravity acceleration and $\rho \leq 1$ is a parameter defined by $\rho=\rho_{2} / \rho_{1}$. The subscript $i=1$ and 2 corresponds to the lower and upper layers of the fluid, respectively (see Figure 8).

It should be noted that in the approximation considered, the vorticity in the layer is proportional to $u_{z}^{i}$, and in the case of no velocity shear, system (37) reduces to the well-known equations of two-layer shallow water [19].

Equations (37) describing two-layer shear flows were studied in [4] where a characteristic function was obtained in the form

$$
\begin{aligned}
\hat{\chi}(k)= & 1-g \int_{0}^{h_{1}} \frac{d z}{\left(u^{1}-k\right)^{2}}-g \int_{h_{1}}^{h_{1}+h_{2}} \frac{d z}{\left(u^{2}-k\right)^{2}} \\
& +(1-\rho) g^{2} \int_{0}^{h_{1}} \frac{d z}{\left(u^{1}-k\right)^{2}} \int_{h_{1}}^{h_{1}+h_{2}} \frac{d z}{\left(u^{2}-k\right)^{2}} .
\end{aligned}
$$

Equation $\hat{\chi}(k)=0$ defines the velocity of propagation of a perturbation in the fluid. In the case of stratified fluid $(\rho<1)$ the characteristic function $\hat{\chi}(k)$ involves a nonlinear term (with multiplication of integrals of the functions $1 /\left(u^{i}-k\right)^{2}$ over the depths of the lower and upper layers). This complicates the analysis and formulation of the hyperbolicity conditions for (37).

Let us consider the following class of flows,

$$
\begin{array}{ll}
u^{1}(t, x, z)=\omega_{1} z+u_{1}=\omega_{1}\left(z-\frac{h_{1}}{2}\right)+\bar{u}_{1}, & z \in\left(0, h_{1}\right), \\
u^{2}(t, x, z)=\omega_{2}\left(z-h_{1}\right)+u_{2}=\omega_{2}\left(z-h_{1}-\frac{h_{2}}{2}\right)+\bar{u}_{2}, & z \in\left(h_{1}, h_{1}+h_{2}\right),
\end{array}
$$

Copyright (C) by SIAM. Unauthorized reproduction of this article is prohibited. 
where, as before, $\omega_{i}(i=1,2)$ are the constant vorticities in the layers, $u_{i}$ are the velocities at the lower boundaries of the layers (at $z=0$ and $z=h_{1}+0$ ), and $\bar{u}_{i}(t, x)$ are the layer-averaged velocities. The corresponding velocity profile is presented in Figure 9 for $\omega_{1}=1, \omega_{2}=1 / 8, h_{1}=h_{2}=1, \bar{u}_{1}=\omega_{1} h_{1} / 2, \bar{u}_{2}=\bar{u}_{1}+0.6$ (solid), and $\bar{u}_{2}=\bar{u}_{1}+0.9$ (dashed line). These profiles differ only in the magnitude of the sliding at the fluid interface.

In this case (37) takes the form

$$
\begin{array}{ll}
\bar{u}_{1 t}+\bar{u}_{1} \bar{u}_{1 x}+\left(g+\frac{\omega_{1}^{2} h_{1}}{4}\right) h_{1 x}+g \rho h_{2 x}=0, & h_{1 t}+\left(h_{1} \bar{u}_{1}\right)_{x}=0, \\
\bar{u}_{2 t}+\bar{u}_{2} \bar{u}_{2 x}+g h_{1 x}+\left(g+\frac{\omega_{2}^{2} h_{2}}{4}\right) h_{2 x}=0, & h_{2 t}+\left(h_{2} \bar{u}_{2}\right)_{x}=0 .
\end{array}
$$

If $\rho=1$ (homogeneous fluid), (40) coincides with system (27) for $N=2$. To study hyperbolicity of (40) we rewrite this system in form (32), where $\mathbf{u}=\left(h_{1}, h_{2}, \bar{u}_{1}, \bar{u}_{2}\right)^{\mathrm{T}}$ is the unknown vector, and $A(\mathbf{u})$ is a matrix of $4 \times 4$. The eigenvalues of $A(\mathbf{u})$ are determined by equations

$$
D(k)=\left(\left(\bar{u}_{1}-k\right)^{2}-\alpha_{1} h_{1}\right)\left(\left(\bar{u}_{2}-k\right)^{2}-\alpha_{2} h_{2}\right)-g^{2} \rho h_{1} h_{2}=0,
$$

where

$$
\alpha_{1}=g+\frac{\omega_{1}^{2} h_{1}}{2}, \quad \alpha_{2}=g+\frac{\omega_{2}^{2} h_{2}}{2} .
$$

System (40) is hyperbolic if (41) has four real roots.

The characteristic velocities $k$ can be directly obtained from equation $\hat{\chi}(k)=$ 0 . Indeed, substituting piecewise linear velocity profile (39) into (38) leads to the following relation,

$$
D(k)=\left(u_{1}-k\right)\left(v_{1}-k\right)\left(u_{2}-k\right)\left(v_{2}-k\right) \hat{\chi}(k),
$$

where $u_{i}$ and $v_{i}$ are the fluid velocities at the lower and upper boundaries of the layers. As was shown before, in the particular case $u_{2}=v_{1}$ (continuous velocity profile) and $\rho=1$ (homogeneous fluid) the considered model is always hyperbolic.

An insightful geometric interpretation of the characteristics proposed by Ovsyannikov [19] for two-layer potential flows $\left(\omega_{i}=0\right)$ can be applied here. We introduce the new variables $p$ and $q$ by the formulas

$$
\bar{u}_{1}-k=p \sqrt{\alpha_{1} h_{1}}, \quad \bar{u}_{2}-k=q \sqrt{\alpha_{2} h_{2}} ;
$$

then (41) can be rewritten in the form

$$
\left(p^{2}-1\right)\left(q^{2}-1\right)=\frac{g^{2} \rho}{\alpha_{1} \alpha_{2}} .
$$

In the $(p, q)$-plane, (43) describes a fourth-order curve with four symmetry axes (see Figure 10). The variables $p$ and $q$ by virtue of (42) are related by

$$
q=p \sqrt{\alpha_{1} / \alpha_{2}}+\left(\bar{u}_{2}-\bar{u}_{1}\right) / \sqrt{\alpha_{2}} .
$$

The number of real roots of (41) is determined by the number of intersections of the curve (43) with the straight line (44). It is clear that the necessary condition for the existence of 4 real roots is the following inequality,

$$
\mu=\sqrt{1-\frac{g^{2} \rho}{\alpha_{1} \alpha_{2}}}>0
$$

Copyright $@$ by SIAM. Unauthorized reproduction of this article is prohibited. 


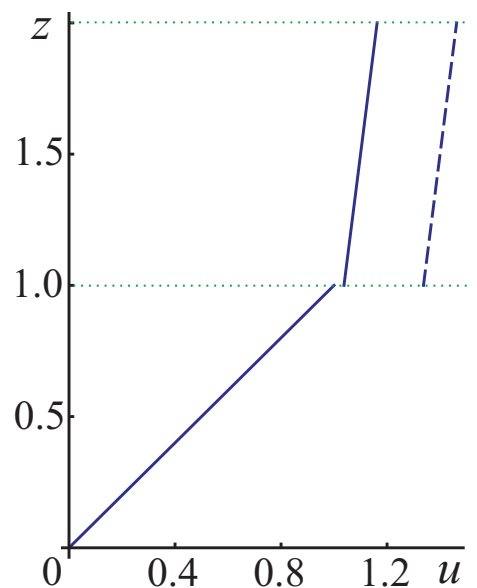

FIG. 9. Piecewise linear velocity profile (39) for $h_{1}=h_{2}=1, \omega_{1}=1, \omega_{2}=1 / 8, \bar{u}_{1}=$ $\omega_{1} h_{1} / 2, \bar{u}_{2}=\bar{u}_{1}+0.6$ (solid), and $\bar{u}_{2}=\bar{u}_{1}+0.9$ (dashed line).

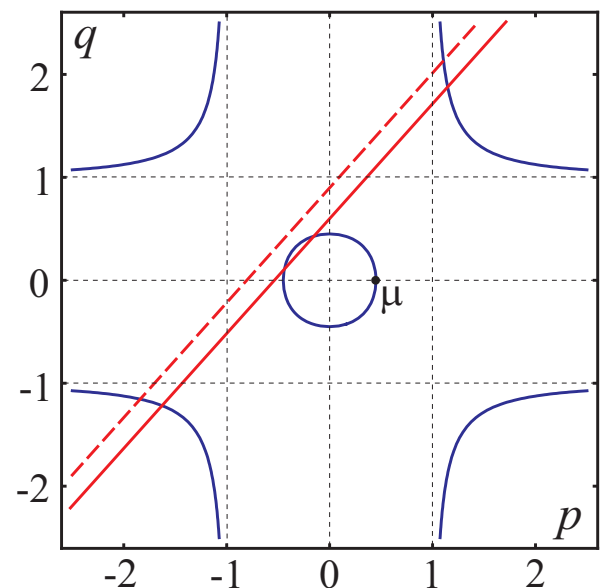

FIG. 10. The curve (43) and the straight lines (44) in the $(p, q)$-plane for the same parameters as in Figure 9 and $g=1, \rho=1$.

which is always fulfilled if $0<\rho \leq 1$ and $\left|\omega_{1}\right|+\left|\omega_{2}\right|>0$. In the case of potential flow $\left(\omega_{i}=0\right)$ the "radius" $\mu$ is $\sqrt{1-\rho}$. Hence, the presence of vorticity improves stability of the two-layer flow. In particular, even for a homogeneous fluid $(\rho=1)$ the flow can be stable, if the sliding between the fluid layers is sufficiently small (see Figures 9 and 10).

5. Conclusion. The classical stability criteria for shear flows (Rayleigh, Fjortoft) are typically obtained for flows between rigid walls. Stability of shear flows with free surface has been much less studied. The main goal of this work was to analyze stability of shallow shear flows with a free surface in terms of hyperbolicity of the nonlinear governing equations. First, we outlined the general hyperbolicity conditions (7) of the Benney equations (4) introduced by Teshukov [23] and Liapidevskii and Teshukov [18]. Further, we have proved in subsection 2.1 that the monotonicity and convexity of the velocity profile are sufficient for the stability of shallow water shear flows with a free surface. This result is also true for the Fjortoft-like velocity profiles (12). Moreover, we presented the class of flows (13) for which the hyperbolicity conditions (7) may be violated. Kinetic formulation (14) of the governing equations allows one to show the analogy between the stability criteria for plasma waves and shear flows.

In the subsequent sections we focus our attention on the multilayer flows with piecewise linear (discontinuous or continuous) velocity profiles described by models (23) and (30). We have revealed some important mathematical properties of the models such as the existence of Riemann invariants (24) and the Hamiltonian structure (28), (29). We have shown that the presence of nonzero vorticity enables one to find multilayer integrable reductions of the Benney system describing shear flows with a class of physically natural continuous velocity profiles, improving the properties of the well-known Zakharov's reductions. For the class of flows with piecewise linear continuous velocity profile we formulated sufficient conditions of stability (36) which are reminiscent of the famous Rayleigh-Fjortoft criterion. The generalization of the results obtained for layered flows of homogeneous fluid to the case of density stratified flows is less obvious. In particular, a continuous velocity profile does not exist. Moreover, the Haantjes tensor (45) does not vanish for system (40) if the density ratio 
$\rho \neq 1$. This mean that the system does not admit Riemann invariants. Nevertheless, we have been able to show that the presence of vorticity has a stabilizing effect on the flow.

Appendix A. The Haantjes tensor: The diagonalizability criterion.

Any strictly hyperbolic system of quasi-linear equations of the type

$$
a_{t}^{i}+v_{j}^{i}(\mathbf{a}) a_{x}^{j}=0, \quad i, j=1, \ldots, M,
$$

can be diagonalized, i.e., can be rewritten in terms of Riemann invariants if and only if the Haantjes tensor [14] constructed in terms of the matrix $v_{j}^{i}(\mathbf{a})$ is identically vanishing [11]. For computing of the Haantjes tensor one calculates first the Nienhuis tensor

$$
N_{j k}^{i}=v_{j}^{p} \partial_{p} v_{k}^{i}-v_{k}^{p} \partial_{p} v_{j}^{i}-v_{p}^{i}\left(\partial_{j} v_{k}^{p}-\partial_{k} v_{j}^{p}\right), \quad \partial_{p} \equiv \partial / \partial a^{p},
$$

and then finally the Haantjes tensor

$$
H_{j k}^{i}=N_{p n}^{i} v_{j}^{p} v_{k}^{n}-N_{j n}^{p} v_{p}^{i} v_{k}^{n}-N_{n k}^{p} v_{p}^{i} v_{j}^{n}+N_{j k}^{p} v_{n}^{i} v_{p}^{n} .
$$

Symbolic computations show that the Haantjes tensor (45) vanishes identically for system (40) if and only if $\rho=1$. This fact justifies the existence of Riemann invariants given explicitly by (24) for the case of a homogeneous fluid.

Acknowledgments. The authors thank IMéRA foundation of Aix-Marseille Université for hospitality. They are also grateful to P. V. Kovtunenko for performing symbolic computations in Appendix A.

\section{REFERENCES}

[1] D. J. Benney, Some properties of long nonlinear waves, Stud. Appl. Math., 52 (1973), pp. 45-50.

[2] L. V. Bogdanov and B. G. Konopelchenko, Symmetry constraints for dispersionless integrable equations and systems of hydrodynamic type, Phys. Lett. A., 330 (2004), pp. 448-459.

[3] J. C. Burns, Long waves in running water, Proc. Cambridge Philos. Soc., 49 (1953), pp. 695-706.

[4] A. A. Chesnokov, On the propagation of long-wave perturbations in a two-layer free-boundary rotational fluid, J. Appl. Mech. Tech. Phys., 45 (2004), pp. 230-238.

[5] A. A. Chesnokov And A. K. Khe, Is Landau damping possible in a shear fluid flow? Stud. Appl. Math., 131 (2013), pp. 343-358.

[6] A. A. Chesnokov And M. V. Pavlov, Reductions of kinetic equations to finite-component systems, Acta Appl. Math., 122 (2012), pp. 367-380.

[7] L. Chumakova, F. E. Manzaque, P. A. Milewsini, R. R. Rosales, E. G. Tabak, and C. V. Turner, Shear instability for stratified hydrostatic flows, Comm. Pure Appl. Math., 62 (2009), pp. 183-197.

[8] L. Chumakova, F. E. Manzaque, P. A. Milewski, R. R. Rosales, E. G. Tabak, and C. V. TuRner, Stability properties and nonlinear mappings of two and three-layer stratified flows, Stud. Appl. Math., 122 (2009), pp. 123-137.

[9] R. C. Davidson, Methods in Nonlinear Plasma Theory, Academic Press, New York, 1972.

[10] P. G. Drazin, Introduction to Hydrodynamic Stability, Cambridge University Press, Cambridge, 2002.

[11] E. V. Ferapontov And S. P. Tsarev, Hydrodynamic type systems, arising in gas chromatography. Riemann invariants and exact solutions, Math. Model., 3 (1991), pp. 82-91 (in Russian).

[12] N. C. Freeman, Simple waves on shear flow: Similarity solutions, J. Fluid Mech., 56 (1972), pp. $257-263$.

[13] S. L. Gavrilyuk and V. M. Teshukov, Linear stability of parallel inviscid flows of shallow water and bubbly fluid, Stud. Appl. Math., 113 (2004), pp. 1-29.

[14] J. HaAntJes, On $X_{m}$-forming sets of eigenvectors, Indag. Math., 17 (1955), pp. 158-162.

[15] E. Yu. Knyazeva And A. A. Chesnokov, Stability criterion of shear fluid flow and the hyperbolicity of the long-wave equations, J. Appl. Mech. Tech. Phys., 53 (2012), pp. 657-663. 
[16] B. A. Kupershmidt And Yu. I. Manin, Equations of long waves with a free surface. II. Hamiltonian structure and higher equations, Funk. Anal. Priloz., 12 (1978), pp. 20-29.

[17] D. R. Lebedev and Yu. I. Manin, Conservation laws and Lax representation of Benney's long wave equations Phys. Lett. A., 74 (1979), pp. 154-156.

[18] V. Yu. Liapidevski and V. M. Teshukov, Mathematical Models of Propagation of Long Waves in a Non-Homogeneous Fluid, Siberian Division of the Russian Academy of Sciences, Novosibirsk, 2000 (in Russian).

[19] L. V. Ovsyannikov, Two-layer shallow-water model, J. Appl. Mech. Tech. Phys., 20 (1979), pp. 127-135.

[20] P. L. SACHDEv, Self-similarity and Beyond: Exact Solutions of Nonlinear Problems, Chapman \& Hall/CRC, Boca Raton, FL, 2000.

[21] T. Stix, The Theory of Plasma Waves, McGraw-Hill, New York, 1962.

[22] J. J. Stoker, Water Waves: The Mathematical Theory with Applications, Interscience, New York, 1957.

[23] V. M. Teshukov, Hyperbolicity of long-wave equations, Dokl. Akad. Nauk., 284 (1985), pp. $555-559$.

[24] V. M. Teshukov, Long waves in an eddying barotropic liquid, J. Appl. Mech. Tech. Phys., 35 (1994) pp. 823-831.

[25] V. Teshukov, G. Russo, and A. Chesnokov, Analytical and numerical solutions of the shallow water equations for 2-D rotational flows, Math. Models Methods Appl. Sci., 14 (2004), pp. 1451-1479.

[26] V. M. Teshukov And M. M. Sterkhova, Characteristic properties of the system of equations of a shear flow with non-monotonic velocity profile, J. Appl. Mech. Tech. Phys., 36 (1995), pp. 367-372.

[27] E. Varley And P. A. Blythe, Long eddies in sheared flows, Stud. Appl. Math., 68 (1983), pp. 103-187.

[28] V. E. Zakharov, Benney equations and quasi-classical approximation in the method of inverse problem, Funk. Anal. Prilozh., 14 (1980), pp. 15-24.

Copyright (c) by SIAM. Unauthorized reproduction of this article is prohibited. 